\title{
Rebecca Stein-Wexler, Sandra L. Wootton-Gorges and M.B. Ozonoff (Eds): Pediatric Orthopedic Imaging
}

\author{
Springer Verlag Berlin Heidelberg, 2015. ISBN: 978-3-642-45380-9
}

\author{
Camilla Russo $^{1} \cdot$ Gianfranco Vallone $^{1}$
}

Published online: 11 June 2016

(C) Springer-Verlag Berlin Heidelberg 2016

This volume is the most recent edition of the book "Pediatric Orthopedic Imaging", first published in 1979. The authors are experts in paediatric imaging with specific reference to orthopaedic diagnosis: Rebecca Stein-Wexler, Sandra L. WoottonGorges and M.B. Ozonoff of the Department of Radiology, University of California Davis in Sacramento. The book is designed for radiology specialists who want to increase their skill in the field of paediatric orthopaedic imaging in relation to both conventional and advanced imaging modalities.

The book consists of 971 pages in 27 chapters, divided into three parts. The first part is dedicated to a brief overview of musculoskeletal imaging strategies, management of radiation exposure in accordance with the principle of radiation protection (justification, optimization and dose limitation) and methods for evaluating normal development. The second part covers focal (congenital, developmental or acquired) musculoskeletal disorders. The chapters are organized according to anatomical region. Before considering pathology, the authors always provide a description of normal anatomy and of most common anatomical variants. The third part focuses on generalized and systemic diseases organized by pathology type. In particular, emphasis is placed on accidental and nonaccidental skeletal trauma and on tumours and tumour-like conditions of bone and soft tissues. The last chapter is dedicated to the orthopaedic procedures that have a great impact on the selection of the most appropriate examination method and on the correct imaging interpretation.

Gianfranco Vallone

gianfranco.vallone2@unina.it

1 Department of Advanced Biomedical Sciences, Università degli Studi di Napoli “Federico II”, Via Pansini, 5, Naples 80131, Italy
The volume contains more than 1,800 illustrations. These are mainly diagnostic images, but there are also many diagrammatic representations of disease pathophysiology and summary tables for rapid consultation.

The text provides accurate descriptions and comparisons of all diagnostic imaging methods, with attention to both traditional and newer radiological procedures focusing on the integration of different techniques in order to optimize diagnostic performance and therefore minimize radiation exposure (a not unimportant matter in younger patients). The authors combine theory and concrete examples, responding to the specific need for a professional approach to radiology, making the book less suitable for nonradiologists. Great emphasis is put on pitfalls and difficulties that might occur in differential diagnosis. The text is globally well-constructed, complete and detailed. The different subjects are discussed clearly, taking into account all the possible imaging procedures. The bibliography contains references to the most recent material, providing a comprehensive review of available scientific literature on the various subjects.

In conclusion, this book covers clearly and comprehensively all the arguments regarding paediatric orthopaedic imaging, giving an exhaustive overview of both focal and generalized musculoskeletal pathology; in this regard it represents a cornerstone in the training of paediatric radiologists. This volume may also stimulate the interest of orthopaedic specialists by providing extensive information in their field of competence. 